\title{
Corrigendum: Peanut Leaf Wilting Estimation From RGB Color Indices and Logistic Models
}

\author{
Sayantan Sarkar ${ }^{1}$, A. Ford Ramsey ${ }^{2}$, Alexandre-Brice Cazenave ${ }^{1}$ and Maria Balota ${ }^{1 *}$ \\ ${ }^{1}$ School of Plant and Environmental Sciences, Virginia Tech, Tidewater AREC, Suffolk, VA, United States, ${ }^{2}$ Department of \\ Agricultural and Applied Economics, Virginia Tech, Blacksburg, VA, United States
}

Keywords: peanut leaf wilting, RGB color space indices, logistic regression, machine learning, high-throughput phenotyping

\section{A Corrigendum on}

Peanut Leaf Wilting Estimation From RGB Color Indices and Logistic Models

by Sarkar, S., Ramsey, A. F., Cazenave, A.-B., and Balota, M. (2021). Front. Plant Sci. 12:658621. doi: $10.3389 / \mathrm{fpls.2021.658621}$

In the original article, there was a mistake in Table 5 as published. There were typos in the text and numbers of the table. The corrected Table 5 appears below:

In the original article, there was an error. There were typos in the equations of Model 1.

OPEN ACCESS

Approved by:

Frontiers Editorial Office,

Frontiers Media SA, Switzerland

${ }^{*}$ Correspondence:

Maria Balota

mbalota@vt.edu

Specialty section:

This article was submitted to Technical Advances in Plant Science,

a section of the journal

Frontiers in Plant Science

Received: 24 November 2021

Accepted: 25 November 2021

Published: 05 January 2022

Citation:

Sarkar S, Ramsey AF, Cazenave A-B and Balota M (2022) Corrigendum: Peanut Leaf Wilting Estimation From

RGB Color Indices and Logistic Models. Front. Plant Sci. 12:821325.

doi: 10.3389/fpls.2021.821325
A correction has been made to Results, Ordinal Logistic Models to Estimate Wilting (Ordinal 0-5 Rating), paragraph 3, Model 1 equations:

Model 1 for proximal RGB images:

$$
\begin{gathered}
P_{0}=\frac{e^{\left(\varepsilon_{a}-11.75\right)}}{1+e^{\left(\varepsilon_{a}-11.75\right)}} \\
P_{1}=\frac{e^{\left(\varepsilon_{a}-7.19\right)}}{1+e^{\left(\varepsilon_{a}-7.19\right)}}-P_{0} \\
P_{2}=\frac{e^{\left(\varepsilon_{a}-4.28\right)}}{1+e^{\left(\varepsilon_{a}-4.28\right)}}-P_{0}-P_{1} \\
P_{3}=1-P_{0}-P_{1}-P_{2}
\end{gathered}
$$

In the original article, there was a mistake in Table 9 as published. There were typos in the numbers of the table. The corrected Table 9 appears below.

The authors apologize for these errors and state that this does not change the scientific conclusions of the article in any way. The original article has been updated. 
TABLE 5 | Wilting accuracy matrix with the number of manually taken wilting scores (2018) on a visual scale at the left and outside the table and the count of image-derived wilting scores in the table.

\begin{tabular}{|c|c|c|c|c|c|c|c|}
\hline \multirow[b]{2}{*}{ Visual wilting score } & \multirow[b]{2}{*}{ Number of manually taken wilting scores } & \multicolumn{6}{|c|}{ Image-derived wilting score (0-5 scale) } \\
\hline & & 0 & 1 & 2 & 3 & 4 & 5 \\
\hline 0 & 4 & 0 & 4 & 0 & 0 & $\bullet$ & $\bullet$ \\
\hline 1 & 72 & 0 & 52 & 20 & 0 & $\bullet$ & $\bullet$ \\
\hline 2 & 65 & 0 & 20 & 41 & 4 & $\bullet$ & $\bullet$ \\
\hline 3 & 26 & 0 & 0 & 20 & 6 & $\bullet$ & $\bullet$ \\
\hline 4 & 0 & $\bullet$ & $\bullet$ & $\bullet$ & $\bullet$ & $\bullet$ & $\bullet$ \\
\hline 5 & 0 & $\bullet$ & $\bullet$ & $\bullet$ & $\bullet$ & $\bullet$ & $\bullet$ \\
\hline Total & 167 & & & & & & \\
\hline Accuracy & $59 \%$ & 0 & $72 \%$ & $63 \%$ & $23 \%$ & $\bullet$ & $\bullet$ \\
\hline Accuracy (second probability method) & $91 \%$ & & & & & & \\
\hline Accuracy (nearest score method) & $99 \%$ & & & & & & \\
\hline
\end{tabular}

\begin{tabular}{|c|c|c|c|c|c|c|c|}
\hline \multirow[b]{2}{*}{ Visual wilting score } & \multirow[b]{2}{*}{ Number of manually taken wilting scores } & \multicolumn{6}{|c|}{ Aerial images } \\
\hline & & 0 & 1 & 2 & 3 & 4 & 5 \\
\hline 0 & 87 & 85 & 0 & 2 & 0 & 0 & 0 \\
\hline 1 & 13 & 0 & 3 & 8 & 1 & 1 & 0 \\
\hline 2 & 27 & 0 & 2 & 13 & 6 & 2 & 0 \\
\hline 3 & 20 & 0 & 0 & 7 & 6 & 6 & 0 \\
\hline 4 & 16 & 0 & 0 & 5 & 3 & 8 & 0 \\
\hline 5 & 5 & 0 & 0 & 1 & 1 & 2 & 1 \\
\hline Total & 168 & & & & & & \\
\hline Accuracy & $69 \%$ & $98 \%$ & $23 \%$ & $48 \%$ & $31 \%$ & $50 \%$ & $20 \%$ \\
\hline Accuracy (second probability method) & $81 \%$ & & & & & & \\
\hline Accuracy (nearest score method) & $90 \%$ & & & & & & \\
\hline
\end{tabular}

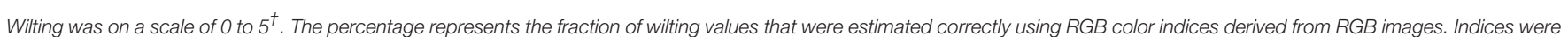
used to estimate leaf wilting using ordinal logistic regression*. The proximal images were taken 11 and 13 weeks after planting (WAP) whereas the aerial images were taken 15 WAP.

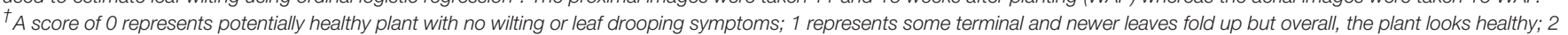
represents almost all leaves fold up and show signs of wilting, lower and older leaves start to fold; 3 represents wilting and drooping shows up on all leaves of the plant, low-moisture effect.

TABLE 9 | Wilting accuracy matrix with the number of manual wilting scores (2019) on a visual scale at the left and outside the table and the count of image-derived wilting scores in the table.

\begin{tabular}{|c|c|c|c|c|c|c|}
\hline \multirow[b]{2}{*}{ Plant water status } & \multicolumn{6}{|c|}{ Estimated turgid vs. wilted plants } \\
\hline & No of plots within each water status & Turgid & Wilted & No of plots within each water status & Turgid & Wilted \\
\hline Turgid & 89 & 82 & 7 & 90 & 86 & 4 \\
\hline Wilted & 78 & 5 & 73 & 78 & 5 & 73 \\
\hline
\end{tabular}

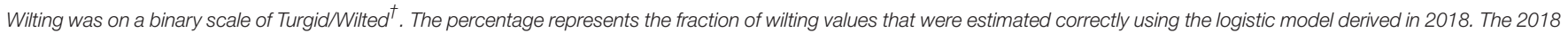
binary models were validated by substituting the RGB color indices ${ }^{\ddagger}$ values derived in 2019 . The proximal and aerial images were taken 15 weeks after planting.

${ }^{\dagger}$ Wilting scores 0 and 1 were rated as turgid and scores above 2 (2 inclusive) were rated as wilted.

$\neq$ Color space indices - Intensity, Hue, Saturation, Lightness, $a^{*}, b^{*}, u^{*}, v^{*}$, green area (GA), greener area (GGA), crop senescence index (CSI). 
Publisher's Note: All claims expressed in this article are solely those of the authors and do not necessarily represent those of their affiliated organizations, or those of the publisher, the editors and the reviewers. Any product that may be evaluated in this article, or claim that may be made by its manufacturer, is not guaranteed or endorsed by the publisher.
Copyright (c) 2022 Sarkar, Ramsey, Cazenave and Balota. This is an open-access article distributed under the terms of the Creative Commons Attribution License (CC $B Y)$. The use, distribution or reproduction in other forums is permitted, provided the original author(s) and the copyright owner(s) are credited and that the original publication in this journal is cited, in accordance with accepted academic practice. No use, distribution or reproduction is permitted which does not comply with these terms. 